\title{
Magnetization-dependent spin Hall effect in a perpendicular magnetized film
}

\author{
T. C. Chuang, ${ }^{1}$ D. Qu $\odot,,^{2, *}$ S. Y. Huang $\odot,{ }^{1, \dagger}$ and S. F. $\mathrm{Lee}^{2}$ \\ ${ }^{1}$ Department of Physics, National Taiwan University, Taipei 10617, Taiwan \\ ${ }^{2}$ Institute of Physics, Academia Sinica, Taipei 11529, Taiwan
}

(Received 21 April 2020; revised 3 August 2020; accepted 10 August 2020; published 31 August 2020)

\begin{abstract}
In the spin Hall effect (SHE), the vector cross-product relations among charge current, spin current, and spin polarization are strictly followed, so that only the in-plane spin accumulation can be induced at the film surface. But, recently this restriction has been lifted by the symmetry breaking of a magnet, where spin polarization can be additionally manipulated by the magnetization directions. In this work, we demonstrated the magnetizationdependent spin-to-charge signals in yttrium iron garnet/Pt/Co/Pt heterostructure. Without the complications in all-metallic structures, we explicitly identified the magnetization-dependent spin Hall effect. We compared its size with the SHE, and further estimated the magnetization-dependent spin Hall angle. Our approach provides an explicit route in exploring the spin-to-charge conversion with arbitrary and controllable orientations of spin polarization, which offers significant advantages in developing electrically controlled energy-efficient spintronic devices.
\end{abstract}

DOI: 10.1103/PhysRevResearch.2.032053

The spin Hall effect (SHE) and inverse spin Hall effect (ISHE), originated from the spin-orbit coupling, are keystones for next-generation spintronic devices [1-3]. The state-ofthe-art spin-orbit torque magnetic random-access memory is based on the efficient charge to spin conversion via the SHE [4]. On the other hand, through the inverse process of the SHE, pure spin current can be efficiently converted into electric current, allowing alternative conversions of heat and light into electricity [5,6]. For the SHE, charge to spin conversion is governed by $\boldsymbol{J}_{\boldsymbol{S}}^{\mathbf{S H E}}=\frac{\hbar}{2 e} \theta_{\mathrm{SH}} \boldsymbol{J}_{\boldsymbol{C}} \times \boldsymbol{\sigma}^{\mathbf{S H E}}$, where the spin current $\boldsymbol{J}_{S}$, charge current $\boldsymbol{J}_{\boldsymbol{C}}$, and spin polarization $\boldsymbol{\sigma}$ are mutually orthogonal. Here, $\hbar$ is the Planck constant, $e$ is the electric charge, and $\theta_{\mathrm{SH}}$ is the spin Hall angle. As a result, an in-plane $\boldsymbol{J}_{\boldsymbol{C}}(x$ axis) can only generate in plane $\boldsymbol{\sigma}(y$ axis) at the film surface, as shown in Fig. 1(a). However, this restriction may hinder the performance of spintronic devices, for example, the in-plane $\sigma$ is not favorable for the field-free spin-orbit torque switching device, which is one of the most important recent advances in spintronics [7]. Intensive researches have shown that additional sources of symmetry breaking, such as geometrically structural asymmetry [8], competing spin current [9], effective exchange field [10,11], or oblique columnar microstructure [12] are necessary.

Strikingly, recent discoveries of spin and charge conversion in magnets suggested that the spin polarization $\sigma$ can be manipulated by the magnetization direction of a magnet [13-18], where one achieves arbitrary spin polarizations at

\footnotetext{
*danru@gate.sinica.edu.tw

†syhuang@phys.ntu.edu.tw

Published by the American Physical Society under the terms of the Creative Commons Attribution 4.0 International license. Further distribution of this work must maintain attribution to the author(s) and the published article's title, journal citation, and DOI.
}

the film surface. The additional spin degree of freedom is not only scientifically interesting but could also lead to significant advances in spintronic applications, for example, the field-free switching devices [19]. The magnetization-dependent spin orientation has been supported by several mechanisms, including the spin rotation effect [14-16], the spin swapping effect [17], and the magnetic spin Hall effect [18], although with different underlying mechanisms, these effects essentially describe the same physical phenomenon, which is the currentinduced unconventional pure spin accumulation due to the time-reversal symmetry breaking of a magnet. Hereafter, we refer to the magnetization-dependent spin Hall effect (MDSHE) as these effects to highlight the crucial role of the magnetization.

One simple microscopic picture to describe the MDSHE is that spins generated from the SHE precess about the magnetization unit vector $\boldsymbol{m}$, and do not dephase rapidly at the interface between a ferromagnetic (FM) and a nonmagnetic (NM) layer [15]. This spin accumulation $\sigma^{\text {MDSHE }}$ is orthogonal to both $\boldsymbol{\sigma}^{\mathrm{SHE}}$ and $\boldsymbol{m}$, and the spin current induced by the MDSHE can be empirically described by

$$
\boldsymbol{J}_{\boldsymbol{S}}^{\mathrm{MDSHE}}=\frac{\hbar}{2 e} \theta_{\mathrm{MDSH}} \boldsymbol{J}_{\boldsymbol{C}} \times\left(\boldsymbol{\sigma}^{\mathrm{MDSHE}} \times \boldsymbol{m}\right) .
$$

Similar to $\theta_{\mathrm{SH}}, \theta_{\mathrm{MDSH}}$ is the magnetization-dependent spin Hall angle, which quantifies the conversion efficiency between spin and charge due to the MDSHE. According to Eq. (1), $\sigma^{\text {MDSHE }}$ can be manipulated by reorienting both $\boldsymbol{J}_{\boldsymbol{C}}$ and $\boldsymbol{m}$ in the FM. When $\boldsymbol{m}$ ( $z$ axis) is out of plane perpendicular to $\boldsymbol{J}_{\boldsymbol{C}}$, $\sigma^{\text {MDSHE }}$ is in plane along the $x$ axis, and when $\boldsymbol{m}$ ( $x$ axis) is in plane along $\boldsymbol{J}_{\boldsymbol{C}}, \boldsymbol{\sigma}^{\mathrm{MDSHE}}$ is out of plane along the $z$ axis, as shown in Figs. 1(b) and 1(c), respectively. Worth to point out, when $\boldsymbol{m}$ ( $y$ axis) is in plane perpendicular to $\boldsymbol{J}_{\boldsymbol{C}}$, it is the anomalous Hall effect (AHE), with spins at the film surface polarized parallel to $\boldsymbol{m}$. 

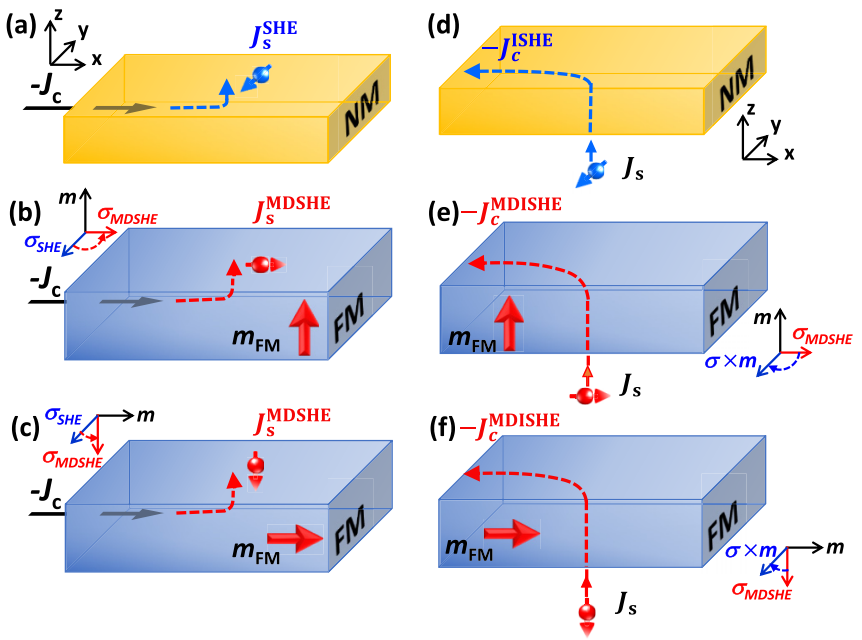

FIG. 1. Schematic drawing of (a) spin Hall effect, (b) magnetization-dependent spin Hall effect in perpendicular magnetized film, (c) magnetization-dependent spin Hall effect in in-plane magnetized film, (d) inverse spin Hall effect, (e) magnetizationdependent inverse spin Hall effect in perpendicular magnetized film, (f) magnetization-dependent inverse spin Hall effect in in-plane magnetized film. For simplicity, one polarization of opposite spins is drawn for the pure spin current.

Notably, by analogy with the ISHE $\left(\boldsymbol{J}_{\boldsymbol{C}}^{\mathrm{ISHE}}=\frac{2 e}{\hbar} \theta_{\mathrm{SH}} \boldsymbol{J}_{\boldsymbol{S}} \times\right.$ $\sigma^{\mathrm{SHE}}$ ), which converts injected spin current back to transverse charge current, as shown in Fig. 1(d), the magnetizationdependent inverse spin Hall effect (MDISHE) converts injected spin current $\boldsymbol{J}_{S}$ back to charge current $\boldsymbol{J}_{\boldsymbol{C}}^{\mathrm{MDISHE}}$ in a direction orthogonal to both $\boldsymbol{J}_{S}$ and $\boldsymbol{\sigma}^{\mathrm{MDSHE}} \times \boldsymbol{m}$, as shown in Figs. 1(e) and 1(f). Thus, reorienting $\boldsymbol{m}$ in the FM controls the direction of the induced charge current. And, the MDISHE can be empirically described by

$$
\boldsymbol{J}_{\boldsymbol{C}}^{\mathrm{MDISHE}}=\frac{2 e}{\hbar} \theta_{\mathrm{MDSH}} \boldsymbol{J}_{S} \times\left(\boldsymbol{\sigma}^{\mathrm{MDSHE}} \times \boldsymbol{m}\right) .
$$

One possible microscopic mechanism for MDISHE is that the spin polarization $\sigma$ rotates about $\boldsymbol{m}$ at the FM/NM interface when entering the magnet, and then being scattered to charge current by the ISHE. It has been suggested that the all-metallic spin-valve structure consisting of one NM spacer layer and two magnets $[13,14,19,20]$, or more complex multilayered systems [15] can be used to investigate the MDSHE and MDISHE. In these heterostructures, a spin-current source and a detector are, respectively, served by two magnets, where the $\boldsymbol{m}$ of one magnet is out of plane and the other is in plane.

Demonstrations of the MDSHE and MDISHE with arbitrary spin accumulation beyond SHE and ISHE initiate new applications for spintronic devices. Materials that show signs of the MDSHE include not only ferromagnet but also antiferromagnet and magnetic Weyl semimetals [18]. However, experimentally the identification of MDSHE and MDISHE remains difficult because of the parasitic effects in all metallicbased heterostructures. For example, it is difficult to electrically quantify and separate various contributions from each magnetic layer when the device is composed of several conducting magnetic layers. Furthermore, when the magnetic layers have moments lie in plane, the contributions from the AHE and planar Hall effect need to be carefully excluded. Moreover, high current density induced contributions, such as the spin-transfer torque effect, thermal effect, and Oersted field also need to be taken into account. Caused by these difficulties, experimentally, the unequivocal establishment of MDSHE and MDISHE still awaits further evidence, not to mention the qualitative or quantitative estimation of the size of these effects. The MDSHE in both the conventional and exotic materials are heavily underexplored.

In this work, by exploiting the spin Seebeck effect (SSE) in a magnetic insulator yttrium iron garnet (YIG) [5], we thermally generate and inject spin current into a perpendicular magnetized $\mathrm{Pt} / \mathrm{Co} / \mathrm{Pt}$ film without the complications in the all-metallic structures. We explicitly identified the MDSHE in the perpendicular magnetized film, quantitatively estimated the $\theta_{\mathrm{MDSH}}$ and $\theta_{\mathrm{SH}}$, and we found the size of the MDSHE is about $3.6 \%$ of the SHE in the $\mathrm{Pt} / \mathrm{Co} / \mathrm{Pt}$ heterostructures.

Two types of samples were fabricated onto the thermally oxidized 0.5 -mm-thick $\mathrm{Si}$ substrate by magnetron sputtering with base pressure better than $10^{-7}$ Torr. One contains the YIG film, on top of which situates the perpendicular magnetized trilayer (PML) $\mathrm{Pt} / \mathrm{Co} / \mathrm{Pt}$, and the other contains only the PML, serving as the reference sample. The 67-nm-thick YIG film was deposited by RF sputtering onto Si substrate at room temperature and then annealed at $800{ }^{\circ} \mathrm{C}$ with flowing oxygen gas by rapid thermal annealing. Our previous report has shown that YIG film deposited on Si substrate is polycrystalline with in-plane magnetic anisotropy and a saturation field of about 50 Oe; see Supplemental Material (SM) [21]. The polycrystalline YIG has the same ability to thermally excite pure spin current as epitaxial YIG [22]. The PML $\operatorname{Pt}(2 \mathrm{~nm}) / \mathrm{Co}(0.5 \mathrm{~nm}) / \mathrm{Pt}(2$ $\mathrm{nm}$ ) were successively deposited onto YIG film or Si substrate by DC sputtering at room temperature. All samples were then patterned by photolithography into a Hall bar structure with three rectangles of $4.55 \mathrm{~mm} \times 0.1 \mathrm{~mm}$. For consistency, we kept the same geometrical and experimental condition for both the SSE and the AHE measurements. The sample was sandwiched between a resistive heater and $\mathrm{Cu}$ heat sink, and a vertical temperature gradient $\nabla T_{\mathrm{z}}=40 \mathrm{~K} / \mathrm{mm}$ was applied. The entire device was placed on top of a stage integrated with two individual magnets providing in-plane or out-ofplane magnetic field. Therefore, we were able to keep the exact alignment between the sample and the magnetic field throughout our measurements.

We first verified the perpendicular magnetic anisotropy (PMA) of the PML on YIG by measuring the anomalous Hall resistance $\left(R_{y}\right)$ [23] under a vertical temperature gradient. Since only the out-of-plane magnetization contributes to the AHE, by measuring $R_{y}$, we kept track of the $\boldsymbol{m}_{\mathbf{P M L}}$ during the entire measurement. The Hall voltage was detected in the $y$ direction when a charge current of $0.1 \mathrm{~mA}$ flew along the $x$ direction, as shown in Fig. 2(a). By sweeping the out-of-plane magnetic field $H_{z}$, we observed a clear AHE hysteresis loop with a coercivity of about 100 Oe, as shown in Fig. 2(b). The square and open loop is the confirmation of a strong PMA. Thus, depending on the history of $H_{z}$, the PML magnetization can be fixed in $+z\left(+m_{\mathrm{PML}}\right)$ or $-z\left(-m_{\mathrm{PML}}\right)$ direction. Importantly, the PMA is robust against in-plane magnetic field perturbations. As shown in Figs. 2(c)-2(f), with 
(a)

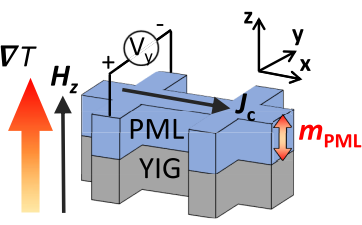

(c)

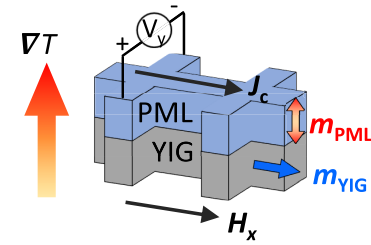

(e)

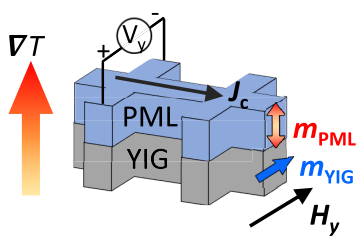

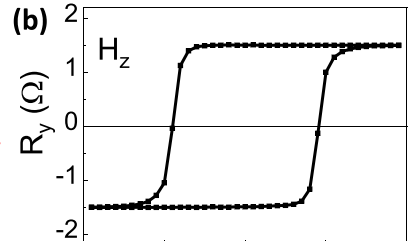

(d)
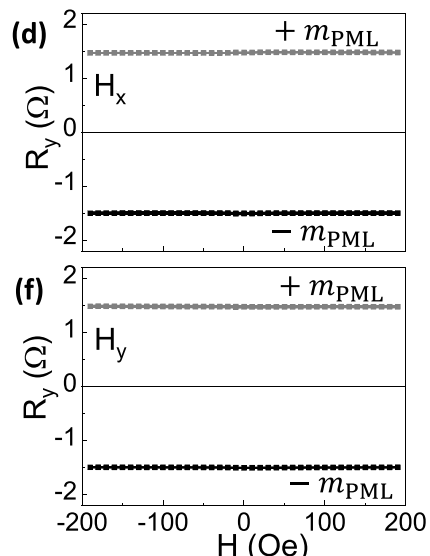

FIG. 2. Schematic drawing of the anomalous Hall measurement geometry featuring the Hall-bar-patterned $\mathrm{YIG}(67) / \mathrm{Pt}(2) /$ $\mathrm{Co}(0.5) / \mathrm{Pt}(2)$ multilayer with charge current flowing along $x$, Hall voltage being measured along $y$, and magnetic field being applied along (a) $z$, (c) $x$, and (e) $y$. (b) Anomalous Hall resistance with field being applied along $z$. Hall resistance with $\boldsymbol{m}_{\text {PML }}$ fixed along $+z$ (gray) or $-z$ (black), while sweeping magnetic field along (e) $x$ and (f) $y$.

an initial magnetization state of PML pointing along $+z$ or $-z$, $R_{y}$ is unchanged under the in-plane magnetic field sweeping along $x\left(H_{x}\right)$ and $y\left(H_{y}\right)$, between +200 and -200 Oe, even for 50 cycles. Only until an in-plane magnetic field larger than 2000 Oe can the $\boldsymbol{m}_{\text {PML }}$ be tilted in plane; see SM [21]. Thus, the magnetization of the PML and YIG film are decoupled and exhibit an orthogonal magnetic alignment. Since the inplane coercivity of PML is much larger than that of YIG, by sweeping the magnetic field in plane up to $\pm 200 \mathrm{Oe}$, we were able to switch the YIG magnetization while keeping the $\boldsymbol{m}_{\text {PML }}$ out of plane. Therefore, the spin polarization of the pure spin current can be controlled by the direction of the YIG magnetization, while the PML magnetization direction can be preset in advance.

We then utilized the SSE to generate and inject spin current from YIG into the PML, as shown in Fig. 3(a). In order to unambiguously observe the MDISHE in the PML and exclude the occurrence of any possible artifacts, we used the configuration in Fig. 1(e), where the polarization of the injected spins and the voltage terminals $\left(V_{x}\right)$ are both along the $x$ direction. Under such configuration, no complications from the ISHE, the AHE, or the anomalous Nernst effect (ANE) are present. The only remaining spin-to-charge signal is the MDISHE in the PML. We set $\boldsymbol{m}_{\mathrm{PML}}$ along $+z\left(+m_{\mathrm{PML}}\right)$ and then swept the magnetic field in the $x$ direction. As mentioned above, within a small field range of $\pm 200 \mathrm{Oe}$, $H_{\mathrm{x}}$ switches magnetization in YIG but not in PML. Our experimental results in this geometry are shown in upper Fig. 3(b). We observed a distinct voltage hysteresis loop with $V_{x}=-0.28 \pm 0.04 \mu \mathrm{V}$ at the positive saturation field. The
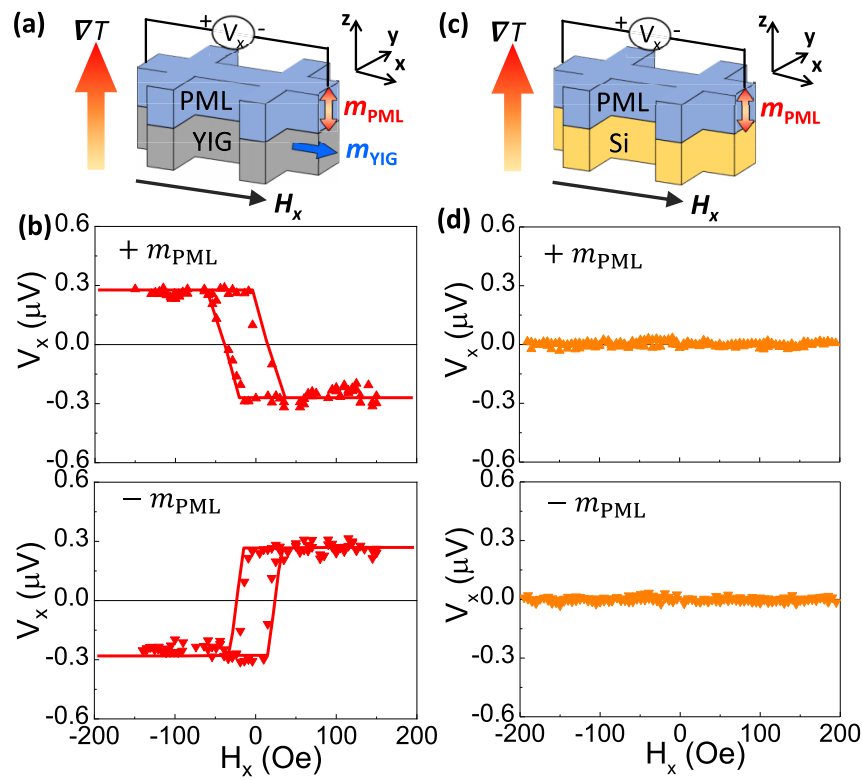

FIG. 3. Schematic drawing of the magnetic inverse spin Hall effect measurement geometry of (a) $\mathrm{Si} / \mathrm{YIG}(67) / \mathrm{Pt}(2) / \mathrm{Co}(0.5) / \mathrm{Pt}(2)$ and (c) $\mathrm{Si} / \mathrm{Pt}(2) / \mathrm{Co}(0.7) / \mathrm{Pt}(2)$ with magnetic field applied and thermal voltage measured both along $x$. Magnetic inverse spin Hall voltage for (b) Si/YIG/Pt(2)/Co(0.5)/Pt(2) and (d) Si/Pt(2)/Co(0.7)/Pt(2) with $\boldsymbol{m}_{\mathrm{PML}}$ fixed along $+z$ (upper) or $-z$ (lower) directions and magnetic field swept along $x$ direction.

saturation field is about $50 \mathrm{Oe}$, corresponding to the saturation field of YIG, which indicates that the thermal voltage is caused by spin-current injection from YIG. Since YIG is an insulator, and any conventional SHE, AHE, and ANE must be zero, voltages measured are entirely attributed to the MDISHE in the attached metallic layers. Most importantly, when $\boldsymbol{m}_{\mathbf{P M L}}$ is flipped to $-z\left(-m_{\mathrm{PML}}\right)$, the voltage hysteresis loop remains essentially the same, but the sign is reversed, as shown in lower Fig. 3(b). With the same spin-current injection, the sign of the detected voltage decisively depends on $\pm m_{\mathrm{PML}}$. This crucial $\boldsymbol{m}$-dependent spin-to-charge conversion is the signature of MDISHE and is consistent with the behavior expected from Eqs. (1) and (2). Thus, we unambiguously demonstrated the MDISHE in the PML. To further validate our finding, we measured the reference sample with only PML on $\mathrm{Si}$, as shown in Fig. 3(c). See SM [21] for details of the PML-only sample. Under the same experimental conditions, the thermal voltage is negligible and independent of the magnetic field. Most importantly, the finite voltage at the saturation field disappears in Fig. 3(d), confirming the signal observed in Fig. 3(b) is a result of spin-current injection from YIG. Worth to point out, keeping the parallel alignment of the Hall bar with the magnetic field is crucial to avoid artifacts, such as the ISHE from YIG or the ANE from the PML.

To quantitatively analyze the spin-orbit interactions, we further compared the MDISHE with the ISHE of the PML. The geometry to observe the ISHE is shown in Fig. 4(a), where the magnetic field is swept along the $y$ direction, and the thermal voltage is measured along the $x$ direction. Within a small in-plane field range of \pm 200 Oe, no ANE is expected due to the strictly fixed out-of-plane $\boldsymbol{m}_{\mathbf{P M L}}$. Therefore, the 

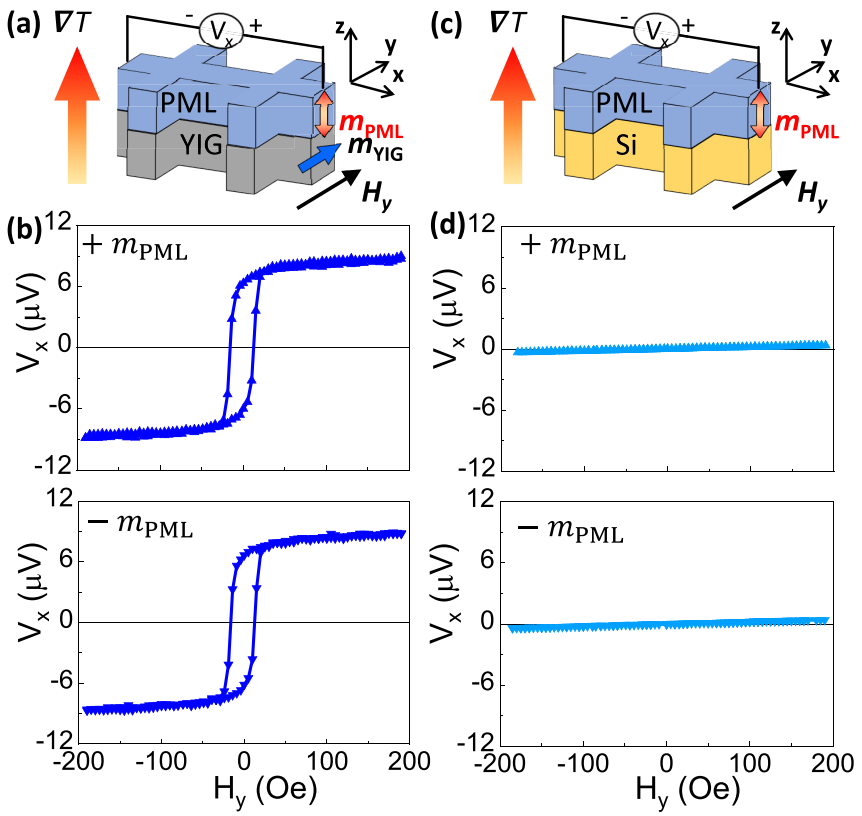

FIG. 4. Schematic drawing of the inverse spin Hall effect measurement geometry of (a) $\mathrm{YIG}(67) / \mathrm{Pt}(2) / \mathrm{Co}(0.5) / \mathrm{Pt}(2)$ and (c) $\mathrm{Pt}(2) / \mathrm{Co}(0.7) / \mathrm{Pt}(2)$ with magnetic field applied along $y$ and thermal voltage measured along $x$. (b) Inverse spin Hall voltage for $\mathrm{YIG} / \mathrm{Pt}(2) / \mathrm{Co}(0.5) / \mathrm{Pt}(2)$ and (d) ordinary Nernst voltage for $\mathrm{Pt}(2) / \mathrm{Co}(0.7) / \mathrm{Pt}(2)$ with $\boldsymbol{m}_{\mathbf{P M L}}$ magnetized in $+z$ (upper) or $-z$ (lower) directions and magnetic field swept along $y$ direction.

$V_{x}=7.83 \pm 0.10 \mu \mathrm{V}$ observed in Fig. 4(b) at the saturation field of 50 Oe is attributed entirely to the ISHE in PML. The sizable ISHE in the PML could be due to the spin-to-charge conversion in the Pt layer, which has been widely used as spin-current generator or detector, the thin Co layer, and the interfacial scatterings. As demonstrated by upper and lower Fig. 4(b), with $\boldsymbol{m}_{\text {PML }}$ pointed to the $+z$ and $-z$ axes, respectively, the observed ISHE voltage is $\boldsymbol{m}$ independent, in sharp contrast to that of the MDISHE. For the reference sample in Fig. 4(c), which consists of only the PML without the YIG spin-current source, under the same experimental conditions, only negligible ordinary Nernst effect was observed with voltage signals being linearly dependent on magnetic field but independent of $\boldsymbol{m}_{\text {PML }}$.

With $\pm m_{\mathrm{PML}}$, the detected signals reversed sign for the MDISHE, but remained unchanged for the ISHE. Comparing the $\left|V_{\text {MDISHE }}\right|=0.28 \pm 0.04 \mu \mathrm{V}$ and $\left|V_{\text {ISHE }}\right|=7.83 \pm$ $0.10 \mu \mathrm{V}$, we found the MDISHE is about $3.6 \%$ of the ISHE, indicating at least $3.6 \%$ spin experiences effective $90^{\circ}$ spin rotation at the interface. From the ISHE voltage we calculated the $\theta_{\mathrm{SH}}[24]$ as

$$
V_{\text {ISHE }}=2[C L \nabla T]\left[\rho(t) \theta_{\mathrm{SH}}\right]\left[\left(\lambda_{\mathrm{SF}} / t\right) \tanh \left(t / 2 \lambda_{\mathrm{SF}}\right)\right] .
$$

Here, $C$ is the spin-injection efficiency, representing the magnetic property of YIG [25] and the spin-mixing conductance at the interface, $L=4.5 \mathrm{~mm}$ is the voltage terminal distance, $\nabla T=40 \mathrm{~K} / \mathrm{mm}$ is the temperature gradient. Under the same experimental geometry, the ISHE for the 4-nm-thick Pt on 67-nm-thick polycrystalline YIG film is about $3.8 \mu \mathrm{V}$; see SM [21]. The resistivity $\rho$ is $24 \mu \Omega \mathrm{cm}$ and $49 \mu \Omega \mathrm{cm}$, and the thickness $t$ is 4 and $4.5 \mathrm{~nm}$, for $\mathrm{Pt}$ and $\mathrm{Pt} / \mathrm{Co} / \mathrm{Pt}$, respectively. Taking $\theta_{\mathrm{SH}}=10 \%$ for $\mathrm{Pt}$, and assuming the same short spindiffusion length $\lambda_{\mathrm{SF}}=10 \mathrm{~nm}$ [26] for both $\mathrm{Pt}$ and $\mathrm{Pt} / \mathrm{Co} / \mathrm{Pt}$, according to Eq. (3), we calculated $\theta_{\mathrm{SH}}$ for $\mathrm{Pt} / \mathrm{Co} / \mathrm{Pt}$ to be approximately $10 \%$. Then, the $\theta_{\mathrm{MDSH}}$ for $\mathrm{Pt} / \mathrm{Co} / \mathrm{Pt}$ is roughly $-0.36 \%$. The negative sign is deduced from Eq. (2). This is a lower-bound estimation. In the ISHE, all three layers of $\mathrm{Pt} / \mathrm{Co} / \mathrm{Pt}$ scatter the spin and contribute to the ISHE signal. For the MDISHE, only spins that pass across the Pt/Co interface experience spin precession about magnetization. Moreover, the magnetic proximity effect in Pt could also result in the spin dephasing at the interface, thus reducing the efficiency of the MDISHE. Also, for a shorter $\lambda_{\mathrm{SF}}$ of $\mathrm{Pt} / \mathrm{Co} / \mathrm{Pt}$, due to the increase of extrinsic scattering, the $\theta_{\mathrm{SH}}$ for the trilayer can be higher, resulting in an even larger $\left|\theta_{\mathrm{MDSH}}\right|$.

The PML used in this work shows both the ISHE and the MDISHE, but there are distinct differences between the SHE/ISHE and the MDSHE/MDISHE in the intrinsic property of each. The SHE is even under time-reversal symmetry, whereas the MDSHE is odd; the SHE in the clean limit is independent, while the MDSHE is proportional to the spinrelaxation time [27]. Therefore, to effectively enhance the $\theta_{\mathrm{MDSH}}$, searching for materials with long spin-relaxation time and interface engineering between FM and NM to minimize the spin dephasing may be the key.

In conclusion, through utilizing the spin Seebeck effect in ferrimagnetic insulator YIG, we injected spin current into the magnetic layer with perpendicular magnetic anisotropy, and unambiguously observed the magnetization-dependent inverse spin Hall effect. Unlike the conventional SHE with the locked orientation of spin polarization, we demonstrated the polarized spin current induced by the MDSHE can be arbitrarily and independently controlled by the magnetization direction of the magnetic layer. We report the MDISHE to be about $3.6 \%$ of the ISHE for $\mathrm{Pt}(2) / \mathrm{Co}(0.5) / \mathrm{Pt}(2)$, indicating $3.6 \%$ of injected spins experience the effective spin rotation. Further calculations show the $\theta_{\mathrm{MDSH}}$ is negative and at least $-0.36 \%$ for $\mathrm{Pt}(2) / \mathrm{Co}(0.5) / \mathrm{Pt}(2)$. Our approach provides a clear-cut and explicit route in exploring materials with controllable current-induced spin accumulations, which can significantly benefit future spintronic devices with different avenues for efficient spin-current generation and applications.

The authors would like to thank C. L. Chien for helpful discussions and H. L. Liang for characterizing the magnetic properties of the YIG film. This study was supported by the Ministry of Science and Technology of Taiwan under Grants No. MOST 106-2628-M-002-015-MY3 and No. MOST 1092123-M-002-002, and by the Academia Sinica under the AS Thematic Project No. AS-TP-107-M04 and iMATE Project No. AS-iMATE-109-11. This study was also partly supported by the National Taiwan University.
[1] M. I. Dyakonov and V. I. Perel, Phys. Lett. A 35, 459 (1971).

[2] J. E. Hirsch, Phys. Rev. Lett. 83, 1834 (1999).
[3] E. Saitoh, M. Ueda, H. Miyajima et al., Appl. Phys. Lett. 88, 182509 (2006). 
[4] L. Liu, C.-F. Pai, Y. Li et al., Science 336, 555 (2012).

[5] K. Uchida, H. Adachi, T. Ota et al., Appl. Phys. Lett. 97, 172505 (2010).

[6] Y.-J. Chen and S.-Y. Huang, Phys. Rev. B 99, 094426 (2019).

[7] I. M. Miron, K. Garello, G. Gaudin et al., Nature (London) 476, 189 (2011)

[8] G. Yu, P. Upadhyaya, Y. Fan et al., Nat. Nanotechnol. 9, 548 (2014).

[9] Q. Ma, Y. Li, D. B. Gopman, Yu. P. Kabanov, R. D. Shull, and C. L. Chien, Phys. Rev. Lett. 120, 117703 (2018).

[10] Y.-C. Lau, D. Betto, K. Rode et al., Nat. Nanotechnol. 11, 758 (2016).

[11] S. Fukami, C. Zhang, S. Dutta Gupta et al., Nat. Mater. 15, 535 (2016).

[12] T. C. Chuang, C. F. Pai, and S. Y. Huang, Phys. Rev. Appl. 11, 061005 (2019).

[13] T. Taniguchi, J. Grollier, and M. D. Stiles, Phys. Rev. Appl. 3, 044001 (2015).

[14] V. P. Amin and M. D. Stiles, Phys. Rev. B 94, 104419 (2016).

[15] A. M. Humphries, T. Wang, E. R. J. Edwards et al., Nat. Commun. 8, 911 (2017).

[16] T. Wang, S. Lendinez, M. B. Jungfleisch et al., Appl. Phys. Lett. 116, 012404 (2020).

[17] C. O. Pauyac, M. Chshiev, A. Manchon, and S. A. Nikolaev, Phys. Rev. Lett. 120, 176802 (2018).
[18] M. Kimata, H. Chen, K. Kondou et al., Nature (London) 565, 627 (2019).

[19] S. C. Baek, V. P. Amin, Y. Oh et al., Nat. Mater. 17, 509 (2018).

[20] J. D. Gibbons, D. MacNeill, R. A. Buhrman, and D. C. Ralph, Phys. Rev. Appl. 9, 064033 (2018).

[21] See Supplemental Material at http://link.aps.org/supplemental/ 10.1103/PhysRevResearch.2.032053 for details on the characterization of YIG thin film, Hall resistance as a function of the magnetic field in $\mathrm{YIG} / \mathrm{Pt} / \mathrm{Co} / \mathrm{Pt}$ and $\mathrm{Pt} / \mathrm{Co} / \mathrm{Pt}$ samples, and the ISHE voltage in YIG/Pt sample.

[22] F.-J. Chang, J. G. Lin, and S.-Y. Huang, Phys. Rev. Mater. 1, 031401(R) (2017).

[23] N. Nagaosa, J. Sinova, S. Onoda, A. H. MacDonald, and N. P. Ong, Rev. Mod. Phys. 82, 1539 (2010).

[24] D. Qu, S. Y. Huang, B. F. Miao, S. X. Huang, and C. L. Chien, Phys. Rev. B 89, 140407(R) (2014).

[25] S. M. Rezende, R. L. Rodríguez-Suárez, R. O. Cunha, A. R. Rodrigues, F. L. A. Machado, G. A. Fonseca Guerra, J. C. Lopez Ortiz, and A. Azevedo, Phys. Rev. B 89, 014416 (2014).

[26] H. L. Wang, C. H. Du, Y. Pu, R. Adur, P. C. Hammel, and F. Y. Yang, Phys. Rev. Lett. 112, 197201 (2014).

[27] R. González-Hernández, L. Šmejkal, K. Výborný et al., arXiv:2002.07073. 